\title{
Chapter 2 \\ Crowdsourcing Coordination: A Review and Research Agenda for Crowdsourcing Coordination Used for Macro-tasks
}

\author{
Sangmi Kim and Lionel P. Robert Jr. $\quad$ Pre-Print Version
}

\begin{abstract}
Crowdsourcing has become a widely accepted approach to leveraging the skills and expertise of others to accomplish work. Despite the potential of crowdsourcing to tackle complex problems, it has often been used to address simple micro-tasks. To tackle more complex macro-tasks, more attention is needed to better comprehend crowd coordination. Crowd coordination is defined as the synchronization of crowd workers in an attempt to direct and align their efforts in pursuit of a shared goal. The goal of this chapter is to advance our understanding of crowd coordination to tackle complex macro-tasks. To accomplish this, we have three objectives. First, we review popular theories of coordination. Second, we examine the current approaches to crowd coordination in the HCI and CSCW literature. Finally, the chapter identifies shortcomings in the literature and proposes a research agenda directed at advancing our understanding of crowd coordination needed to address complex macro-tasks.
\end{abstract}

\subsection{Introduction}

Crowdsourcing has become a widely accepted approach to leveraging the skills and expertise of others to accomplish work (Robert and Romero 2015, 2017). Crowdsourcing has many definitions but was first defined by Jeff Howe as the outsourcing of work to a crowd (Howe 2006). Typical modern definitions of crowdsourcing involve two attributes: (1) a crowd, or group of people, and (2) online work. Crowdsourcing platforms such as Mechanical Turk (http://www.mturk.com) and CrowdFlower (http://www.crowdflower.com) attract large groups of people who can work online via these digital platforms. These platforms and the people who work on them (i.e., crowd workers) provide access to a wealth of knowledge and expertise that can be leveraged to tackle complex problems.

\footnotetext{
S. Kim $(\varangle) \cdot$ L. P. Robert

University of Michigan School of Information, Ann Arbor, USA

e-mail: Sangmik@umich.edu
}

\section{P. Robert}

e-mail: lprobert@umich.edu 
Despite the potential of crowdsourcing to tackle complex problems, it has often been used to address rather simple micro-tasks. Micro-tasks are standalone simple tasks that do not require the coordination of work among individuals (Schmitz and Lykourentzou 2018). To tackle more complex problems, crowdsourcing must address macro-tasking. Macro-tasking can be described as complex crowd work that is sometimes but not always decomposable to micro-tasks (Schmitz and Lykourentzou 2018). Crowdsourcing macro-tasks is more challenging than crowdsourcing micro-tasks. Macro-tasking requires work processes needed to tackle complex problem-solving involving activities such as the generation and integration of diverse ideas along with group decision-making. Macro-tasking requires crowd workers to coordinate in order to both divide their labor and aggregate the outputs of their labor.

In the human-computer interaction/computer-supported cooperative work ( $\mathrm{HCl} / \mathrm{CSCW}$ ) fields, crowd coordination is typically handled by the requestor and results in micro-tasking. Requestors divide and assign work prior to any crowd involvement and in many cases the work is never aggregated. Unfortunately, this approach to crowd coordination limits the potential of crowds to solve complex problems and reach their full potential.

Consider the following scenario: An organization wants to use crowdsourcing to identify its next new product. The organization puts forth a call to the public for new ideas and gives a specific deadline. The organization receives many great ideas and asks the crowd to vote on the best idea for a new product. The votes are tallied and the winner is announced. This approach to crowdsourcing is oriented toward microtasking. The work process is reasonably well formulated and easy to understand by all crowd workers. Although the outcome might not be predictable, the work process is very predictable. The crowdsourcing tasks require little interaction or dependence among crowd workers, so coordination is of little importance.

Now consider a different scenario: An organization wants to crowdsource the development of the marketing plan for this new product. Because there are many ways to accomplish this task, the work is not easily nor reasonably well formulated. Both the work process and the outcome are not as predictable as in the last scenario. Because the crowd is expected to produce one marketing plan, the crowd workers must decide how the work is to be divided and how or whether the work needs to be aggregated. To accomplish this task, crowd workers need to work together. This approach to crowdsourcing is oriented toward macro-tasking and requires interaction and greater dependence among crowd workers; therefore, coordination is of the utmost importance. Clearly, to fully leverage crowdsourcing, more work is needed on coordinating the crowdsourcing of macro-tasks.

There are many definitions of coordination (Robert 2016). For the sake of clarity, this chapter defines coordination generally as:

The synchronization of individuals in an attempt to direct and align their efforts in pursuit of a shared goal.

And crowd coordination specifically as:

The synchronization of crowd workers in an attempt to direct and align their efforts in pursuit of a shared goal. 
The goal of this chapter is to advance our understanding of macro-tasking in crowdsourcing by addressing issues related to coordination. To accomplish this, we have three objectives. First, we review popular and recent theories of coordination across organizational and computer science. Specifically, we present and discuss transactive memory systems (TMS), coordination theory, role-based coordination, relational coordination, stigmergic coordination, and an integrative model of coordination. Second, we examine the $\mathrm{HCI}$ and $\mathrm{CSCW}$ studies on coordination in macrotasking and categorize these approaches into one or more of the previously presented theories of coordination. Although prior studies on coordination in crowdsourcing have focused primarily on micro-tasking, attention is shifting toward macro-tasking, as seen by a small but fast-growing set of HCI/CSCW articles on the topic. Last, we propose a research agenda based on the review of coordination theories and prior $\mathrm{HCI}$ and $\mathrm{CSCW}$ work on coordination in macro-tasking.

\subsection{Background}

\subsubsection{Coordination in Micro-tasking Versus Macro-tasking in Crowdsourcing}

The first question one might ask is this: What makes coordinating macro-tasks so different from coordinating micro-tasks? Macro-tasks require much more coordination among workers than micro-tasks, for several reasons. Many micro-tasks are independent individual decomposed tasks assigned to individuals. Standalone independent micro-tasks require little or no coordination among crowd members. However, in many cases, macro-tasks cannot be decomposed to the level of a single individual and require more than one person to perform the work. The interdependent nature of macro-tasking requires coordination among crowd workers. In addition, macrotasks that can be decomposed are likely to be decomposed by the crowd and not the requestor. Both the decomposition of macro-tasks and the eventual aggregation of micro-tasks require coordination among crowd members.

\subsubsection{Theories of Coordination}

\subsubsection{Transactive Memory System}

What is it? A transactive memory system (TMS) is a way of coordinating work that relies on members of a collective to know who knows what in that collective. This is accomplished in part by sharing or dividing the cognitive labor across the collective (Brandon and Hollingshead 2004; Wegner 1987). Research linking TMS to better coordination and ultimately performance has been conducted across a wide 
and diverse set of fields including information systems, organizational behavior, psychology, and communications (Ren and Argote 2011). More specifically, the coordination benefits of TMS have led to emergent and adaptive team behaviors, allowing for effective and implicit communication (Marques-Quinteiro et al. 2013). TMS has proved to be an invaluable approach to team coordination.

How does it work? TMS effectiveness relies on five key elements. First, each member of the collective should hold unique specialized knowledge. Second, members of the collective should share a cognitive map of the distribution of this specialized knowledge across the team. Three, task responsibilities should be assigned to each member of the collective based on their specialized knowledge (Brandon and Hollingshead 2004; Moreland 1999). Four, members of the collective should trust that each member is competent in his or her knowledge domain and assigned task responsibilities (Austin 2003; Lewis 2003). If members of the collective do not trust one another they will be less likely to rely on one another's expertise. Five, members of the collective must communicate with one another to leverage each person's expertise (Choi et al. 2010). Communication allows for the sharing of specialized knowledge, which is essential for leveraging expertise across the collective.

\section{Transaction Memory System Key Elements}

- Specialized knowledge among members

- Shared cognitive map of specialized knowledge

- Task responsibilities based on specialized knowledge

- Members who trust one another's specialized knowledge

- Members who share their specialized knowledge

Potential Benefits for Macro-tasking: TMS allows for coordination among crowd workers through implicit communication. This reduces the overhead associated with explicit communication. TMS can also be used to organize and assign tasks. As new work requirements emerge they are automatically assigned to crowd workers based on their knowledge specialization.

Potential Drawbacks for Macro-tasking: Crowds should have either a shared work history to develop a TMS or some way to communicate who knows what in a crowd. Developing a TMS can take time that crowd workers may not have. Platforms can be designed to communicate who knows what in a crowd. But it could be problematic for existing crowd workers to keep track of who knows what with regard to departing and incoming members.

\subsubsection{Coordination Theory}

What is it? Coordination theory is one of the most popular approaches to understanding coordination (Crowston et al. 2006). The theory defines coordination as the management of "dependencies between activities" (Malone and Crowston 1994, p. 90). One of the distinctive applications of the coordination theory is the use of coordination mechanisms that are based on the type of dependencies among tasks for 
designing collaborative systems (e.g., Andres and Zmud 2002; Strode et al. 2012). Malone and Crowston (1994) introduced ways to analyze coordination in terms of actors, interdependent tasks that are performed by the actors, and resources that are required for completing those tasks. Based on their analysis, coordination problems that arise from the dependencies among tasks, actors, and resources are identified and solved by deploying appropriate coordination mechanisms.

How does it work? Several aspects of coordination theory make it distinct from other theories of coordination. First, it draws attention to the dependencies among tasks rather than among individuals or organizational units (Crowston et al. 2006). Instead of understanding coordination in terms of how people who perform the task relate to one another, this theory views coordination in terms of how one task is related to another task. Second, it identifies and categorizes types of dependencies among activities. This provides clarity as to possible implications associated with specific dependencies. Finally, this theory allows for the modeling of coordination to make it easier to understand the effects of assignments and reassignments of activities needed to complete tasks (Crowston 1994). This allows people to understand the implications of adding or removing members of the collective relative to that change's impact on coordination. However, recent work highlighted the limitations of coordination theory for coordinating crowd work (Retelny et al. 2017).

\section{Coordination Theory Key Elements}

- Identify tasks

- Identify and categorize dependencies among tasks

- Employ appropriate mechanism per dependency type

Potential Benefits for Macro-tasking: Coordination theory allows for the identification and removal of potential barriers to accomplishing crowd work. The workflow plans derived from coordination theory not only provide guidance needed to accomplish work but also a shared communication medium to facilitate a common understanding among crowd workers.

Potential Drawbacks for Macro-tasking: Coordination theory relies heavily on a person or group to pre-plan the work, which is less useful when task requirements are not known or task requirements are emergent and change over time. For example, at least one study has found evidence of this limitation as it relates to crowdsourcing complex adaptive work (see Retelny et al. 2017).

\subsubsection{Role-Based Coordination Theory}

What is it? Role-based coordination relies on roles or a set of expectations associated with a position to organize and perform work (Bechky 2006). Roles constitute both expected activities and their associated responsibilities. Roles have long been viewed in organizations as the basic unit of coordination (Okhuysen and Bechky 2009). Role-based coordination does not rely on specific individuals, which has proved in some cases to be effective for complex and interdependent crowd work with transit membership (e.g., Valentine and Edmondson 2014). 
How does it work? Typically, role-based coordination theories assert that work can be organized by assigning roles to individuals and holding them accountable for the responsibilities associated with their roles. Structure is used to coordinate work across roles and is determined by the relationships among roles within some boundary. Structure can be viewed as either a centralized hierarchical structure or a decentralized flat structure. Role-based coordination theories accomplish work by defining and assigning roles to individuals and ensuring that these roles are structured in a way that best supports the work needed to be done.

\section{Role-Based Coordination Theory Key Elements}

- Role definition

- Role assignment

- Role structure

- Role accountability

Potential Benefits for Macro-tasking: Role-based coordination does not rely on specific individuals to accomplish work but instead relies on roles. Reliance on roles promotes a plug-and-play structure that allows crowd workers to move in and out of the crowd with minimal disruption to work.

Potential Drawbacks for Macro-tasking: Role-based coordination requires someone to create the roles and their corresponding responsibilities. That being the case, it is not clear who would create new roles when needed. This becomes problematic when task requirements are emergent and change over time.

\subsubsection{Relational Coordination Theory}

What is it? Relational coordination theory asserts that a core facilitator of effective work is the quality of interactions among workers (Gittell 2002, 2011). According to Gittell (2002), the quality of interactions is based on effective communications and strong relationships. The underlying logic is that coordination involves both task interdependencies and the interactions among people involved in those tasks. Therefore, higher quality interactions among people involved in those tasks are likely to enhance coordination and lead to better performance (Gittell 2011). According to relational coordination theorists, high-quality relationships are especially important to achieving better performance when work is complex, interdependent, and timeconstrained (Faraj and Xiao 2006; Gittell 2002, 2006, 2011). The importance of the relationships among employees has been supported by several observations in organizational settings (e.g., Adler et al. 2008).

How does it work? Relational coordination theory views coordination as "a mutually reinforcing process of interaction between communication and relationships carried out for the purpose of task integration" (Gittell 2002, p. 301). Relational coordination theory describes relationship in terms of three dimensions: shared goals, shared knowledge, and mutual respect. The theory describes communication in four dimensions: frequency, timeliness, accuracy, and problem-solving focus (Gittell 2002, 
2006). Relational coordination occurs when work is coordinated "through highquality communication, supported by relationships of shared goals, shared knowledge, and mutual respect" (Gittell 2016, p. 11). This indicates that collectives who have more frequent, timely, accurate, and problem-solving-focused communication can be expected to coordinate more effectively and ultimately perform better by having shared goals, shared knowledge, and mutual respect.

\section{Relational Coordination Theory Key Elements}

- Relationships

- Shared goals

- Shared knowledge

- Mutual respect

- Communication

- Frequent

- Timely

- Accurate

- Problem-solving focus

Potential Benefits for Macro-tasking: Coordination via high-quality relationships is very flexible and robust, allowing crowds to adapt to new or emergent task requirements. It relies less on formal planning and more on the possibility of informal planning done by the crowd itself.

Potential Drawbacks for Macro-tasking: It takes time to develop high-quality relationships among crowd workers. However, it is unclear whether current crowdsourcing platforms are designed to support the development of high-quality relationships among crowd workers.

\subsubsection{Stigmergic Coordination Theory}

What is it? Stigmergic coordination can be described as coordination that occurs through changes in a shared or collective work product (Rezgui and Crowston 2018). The concept of stigmergy is derived from entomologists' observations of social insects. Insects such as ants and termites leave traces (e.g., pheromones) while performing work, and such traces stimulate other insects to take subsequent actions (Heylighen 2015; Khuong et al. 2016). Examples of stigmergic coordination on the part of insects include termites building and repairing nests, and ants finding the shortest route to food (Heylighen 2016; Khuong et al. 2016). The concept of stigmergy has influenced the design of collaborative action such as free open-source software development (Bolici et al. 2009, 2016), multi-agent systems (e.g., Valckenaers et al. 2004) and collective robotics (e.g., Holland and Melhuish 1999). These areas have applied the stigmergic coordination approach to the need for coordinating in dynamic and emergent environments without direct communication between workers and agents. 
How does it work? Members of a given collective not only perform work but also leave traces of their work. This requires ensuring that those traces are visible to other members. Those other members interpret those traces to determine what has already been done. Based on this, and their knowledge of what has to eventually be done, they determine the work that needs to be done next. Finally, as they are performing their own work they leave traces behind for other members. The stigmergic process of coordination occurs across many tasks done by many workers. As a result, stigmergic coordination can occur without direct and explicit interactions among members of a collective (Heylighen 2016). Stigmergic coordination seems to operate, in part, based on the development of shared work norms and practices normally associated with communities of practice (Lave 1991, 2009; Lave and Wenger 1991), derived somewhat from Suchman's (1987) work on situated action.

\section{Stigmergic Coordination Theory Key Elements}

- Create traces

- Interpret traces

- Determine future actions based on traces

Potential Benefits for Macro-tasking: Stigmergic coordination relies on distribution cognition, which allows the crowd to self-organize. There is low reliance on specific individuals to accomplish or plan the work. This provides a relative plug-and-play structure for crowd workers from the same community of practice (i.e., shared work norms). Stigmergic coordination employs informal planning that is flexible, robust, and adaptive to new or emergent task requirements.

Potential Drawbacks for Macro-tasking: Crowd workers must share a common set of work norms and practices. Therefore, the plug-and-play structure only applies to members of the same or similar work collectives. In fact, stigmergic coordination might be the worst coordination approach when workers do not share a common set of work norms and practices. As such, it limits the potential set of crowd workers available to recruit from.

\subsubsection{Integrative Coordination Framework}

What is it? The integrative framework was put forth by Okhuysen and Bechky (2009), in part to help identify coordination mechanisms. Based on their literature review on coordination they identified five types of coordination mechanisms (plans and rules, objects and representations, roles, routines, and proximity) and three conditions needed for coordination (accountability, predictability, and a common understanding). Generally, Okhuysen and Bechky's (2009) integrative framework asserts that the five types of coordination mechanisms promote coordination through supporting one or more of the three conditions. Specifically, their framework identifies which coordination mechanisms support which conditions.

How does it work? The integrative framework promotes coordination by identifying the types of mechanisms needed. If one assumes that accountability, predictability, 
and a common understanding are needed, then one could ensure that at least one mechanism is chosen to support each of them. Likewise, if coordination was still a problem, more mechanisms could be employed to help buttress a particular condition. For example, if collectives were struggling with accountability, the integrative framework could help to identify a mechanism that could be employed to improve accountability.

\section{Conditions}

Accountability: Accountability describes who is responsible for specific tasks and elements of those tasks. Making clear and visible who is in charge of which tasks promotes the awareness of each person's interdependence and responsibility, and the development of trust, which is in turn expected to contribute to coordinated actions in a collective. Accountability in the integrative framework includes the means that are created through informal and emergent interactions such as side conversations. Plans, rules, and objects can serve as the scaffolding that links tasks with people who are responsible for them. Roles, routines, and visibility also support continual monitoring, updating, and hand-offs among workers.

Predictability: Predictability explains workers' understanding of what subtasks constitute larger tasks in what sequence and what activities must be performed to accomplish each task. Predictability is essential for coordination because it highlights the anticipation of subsequent tasks and related actions of others and allows workers to adjust their work to others' work and perform their work accordingly. Plans and objects are the coordination mechanisms that create predictability by determining what tasks need to be completed. Familiarity and routines also enhance predictability by providing information on other workers' preferences with regard to the work. Common understanding: Common understanding is a shared knowledge among workers about what the whole completed work is like, including goals and objectives and how it is accomplished. Plans and rules create a common understanding of the whole interdependent task and the process, facilitating better coordination. Routines and familiarity help workers become familiar with the ways the different parts of the work are put together to create the whole. In addition, objects and roles develop a common perspective through sharing and learning different activities to complete tasks.

\section{Coordination Mechanisms}

Plans and rules: As one of the fundamental elements of coordination, "plans and rules" refers to a set of elements that define relationships among tasks, workers, and other units of organizations. Among the functions of plans and rules is defining responsibility for tasks. Coordination by plans and rules enables people to decide what (subsequent) actions to take and what choices to make among the alternatives to complete tasks.

Objects and representations: The effective use of objects, representations, and technologies helps in coordinating work by providing information that is important to accomplish tasks (direct information-sharing). For example, boundary objects (e.g., data spreadsheets) are necessary to communicate problems to solve, ideas, and activities across teams. Also, a representative map or matrix of tasks and responsibilities 
(scaffolding) serves as a frame that reminds people of what tasks to do, the actors in charge of each task, the alignment of tasks among workers, and the progress of work (acknowledging and aligning work).

Roles: Roles can function as a coordination mechanism in two ways. While representing sets of responsibilities and activities of an actor who occupies the position, roles at once allow for redefining the responsibilities to adapt to the emergent status of work (monitoring and updating). This process of defining roles allows for $\mathrm{cre}$ ating a common perspective. Under common understandings about responsibilities, substitution can be easily done.

Routines: In more traditional organizational contexts, "routines" refers to "repeated patterns of behavior that are bound by rules and customs" (Feldman 2000, p. 611). In contrast, the current literature defines "routines" as ways to reflect "social meaning and social interaction ... embedded within them" (Okhuysen and Bechky 2009, p. 477).

Proximity: "Proximity" refers to coordination based on factors often associated with physical distance. These factors include visibility and familiarity. "Visibility" refers to the ability to see what others are doing, which is often associated with collocation but not necessarily a requirement of collocation. "Familiarity" refers to the ability to rely on prior relationships with others to facilitate the coordination of actions. Once again, familiarity has often been associated with collocation but is not necessarily a requirement of collocation.

\section{Integrative Coordination Framework Key Elements}

- Conditions

- Accountability

- Predictability

- Common understanding

- Coordination mechanisms

- Plans and rules

- Objects and representations

- Roles

- Routines

- Proximity

Potential Benefits for Macro-tasking: Because the integrative perspective entails both formal and emergent processes of coordination, the development of the coordination mechanisms and conditions promotes diverse coordination activities. This includes the explanation of a range of coordination procedures and tasks, from defining problems and tasks to completing and handing off tasks.

Potential Drawbacks for Macro-tasking: Establishing such mechanisms and conditions might require a specific set of personnel, which would be expected to take enough time to develop alternative formal and informal patterns of coordinated activities. 


\subsection{Recent Studies on Coordination in Macro-tasking Crowdsourcing}

\subsubsection{Search Methods}

To review recent studies of coordination in macro-tasking, we first employed the academic search engine Google Scholar, entering the search keywords "microtask," "coordination," and "crowdsourcing." We conducted the search in August 2018 and the results showed 60 articles. We read abstracts of the articles and evaluated whether to include the articles in the literature review based on the following inclusion criteria: (1) the article addressed issues about coordination for macro-tasking or (2) the article suggested and tested empirical ideas or designs of macro-task crowdsourcing. We excluded review papers, textbook-type books, patent applications, and articles published in non-English venues. Eight studies met all the criteria from the initial search. Additionally, we traced back some of the initial search results. This was because we found that some studies had been influencing the literature in macro-tasking coordination but had not shown up through our keyword search. For example, Kittur et al. (2011) and Kulkarni et al. (2012) were heavily cited as exemplar of investigating coordination problems of macro-tasking but didn't appear in the initial search results. As a result, we identified a total of ten studies for the literature review.

\subsubsection{Approaches Used to Coordinate Crowdsourcing Macro-tasks}

We reviewed all the papers to identify which coordination theories and which of the five mechanisms were employed. To do this, we first grasped the main ideas and assumptions behind each coordination theory. We used these to make distinctions among them. Then we read and reviewed each study independently and discussed which theory best represented each study's approach to coordination and whether it relied on one of the five mechanisms.

Most studies could be placed within the coordination theory approach (see Table 2.1). These studies typically focused on identifying and managing various dependencies among tasks, roles, and workers. To identify and manage dependencies these studies leveraged various techniques and tools. For example, to understand dependencies at the task level, Kittur et al. (2011) and Kulkarni et al. (2012) proposed systems that displayed plans for the work, including the sequence and the structure of work in units of subtasks. Also, to coordinate available competent workers, Haas et al. (2015) and Schmitz and Lykourentzou (2018) devised systems to model the structure of work by workers' level of skills and expertise. It appears that many HCI and CSCW researchers have addressed issues of coordination in macro-tasking, exploring the ideas best represented by coordination theory. 
Table 2.1 Literature review based on coordination theories

\begin{tabular}{|c|c|c|c|c|c|}
\hline & $\begin{array}{l}\text { Transactive } \\
\text { memory } \\
\text { system }\end{array}$ & $\begin{array}{l}\text { Coordination } \\
\text { theory }\end{array}$ & $\begin{array}{l}\text { Role-based } \\
\text { coordination }\end{array}$ & $\begin{array}{l}\text { Stigmergic } \\
\text { coordination }\end{array}$ & $\begin{array}{l}\text { Relational } \\
\text { coordination }\end{array}$ \\
\hline $\begin{array}{l}\text { Kittur et al. } \\
\text { (2011) } \\
\text { (Crowd- } \\
\text { forge) }\end{array}$ & & $X$ & & & \\
\hline $\begin{array}{l}\text { Kulkarni } \\
\text { et al. (2012) } \\
\text { (Turkomatic) }\end{array}$ & & $X$ & & & \\
\hline $\begin{array}{l}\text { Haas et al. } \\
\text { (2015) } \\
\text { (Argonaut) }\end{array}$ & & $X$ & $X$ & & \\
\hline $\begin{array}{l}\text { Teevan et al. } \\
\text { (2016) } \\
\text { (Microwriter) }\end{array}$ & & $X$ & & & \\
\hline $\begin{array}{l}\text { Kim et al. } \\
(2017) \\
\text { (Mechanical } \\
\text { novel) }\end{array}$ & & $X$ & & & \\
\hline $\begin{array}{l}\text { Retelny et al. } \\
\text { (2017) (No } \\
\text { workflow) }\end{array}$ & & $X$ & & & \\
\hline $\begin{array}{l}\text { Salehi et al. } \\
\text { (2017) } \\
\text { (Huddler) }\end{array}$ & & $X$ & & & $X$ \\
\hline $\begin{array}{l}\text { Valentine } \\
\text { et al. (2017) } \\
\text { (Flash } \\
\text { organization) }\end{array}$ & & & $X$ & & \\
\hline $\begin{array}{l}\text { Kaur et al. } \\
\text { (2018) } \\
\text { (Vocabulary) }\end{array}$ & & $X$ & & & \\
\hline $\begin{array}{l}\text { Schmitz and } \\
\text { Lykourent- } \\
\text { zou (2018) } \\
\text { (Task } \\
\text { assignment } \\
\text { and } \\
\text { sequencing) }\end{array}$ & & $X$ & & & \\
\hline
\end{tabular}


The second most used theory was role-based coordination. We found several studies that employed role-based coordination. These studies typically created a structure of roles and responsibilities for those roles and assigned qualified workers to each role to achieve goals. For example, Valentine et al. (2017) first built a hierarchical structure of roles based on tasks and activities using the role-based coordination theory. This study is in line with previous studies on scaffolding structures of roles in emergent coordination contexts. This includes an emergency unit of a university hospital (Valentine and Edmondson 2014) and emergent student team projects (Retelny et al. 2014; Valentine et al. 2017). We found no studies employing TMS or stigmergic coordination.

Regarding relational coordination, Salehi et al. (2017) study aligned with the relational coordination approach. The authors identified that familiarity among workers was an advantageous condition in performing tasks for distributed crowds. Specifically, when teaming workers up, they accounted for familiarity (e.g., history of collaborations with other members) in addition to availability. They also provided an instant communication channel and collaborative writing platform to support collaboration. The results indicated that the workers working with familiar teammates performed better, knowing well other team members' strengths and work processes. This study was not conducted in the same context as the face-to-face organization interaction that extant research in relational coordination has considered. However, by convening workers who were familiar with one another and leveraging their shared knowledge with the use of proper communication tools, the study successfully examined the effectiveness of relational communication.

In summary, it appears that scholars are overwhelmingly employing coordination theory to explore ways to handle macro-tasking in crowdsourcing. Role-based coordination is a distant second, followed by the relational coordination theory. None of the studies employed TMS or stigmergic approaches. Nonetheless, the literature base is quite nascent, with just two papers before 2015 (in 2011 and 2012) and more than half published in 2017 or 2018.

\subsubsection{Coordination Mechanisms for Crowdsourcing Macro-tasks}

\subsubsection{Evolving Plans and Rules}

Plans and rules have been employed to help identify what tasks need to be completed and to assign crowd workers task responsibilities. Especially in the macro-tasking context, plans and rules for crowd workers should evolve to actively react to changes as work progresses. For example, Kulkarni et al. (2012) proposed Turkomatic, a realtime editable workflow that can be formed by crowds. Turkomatic was developed to allow workers to breakdown complex problems into smaller tasks. Kim et al. (2017) suggested a reflect-and-revise technique with which crowds could work on solving 
complex problems such as story-writing. Emphasizing the importance of higher level goals for complex and open-ended work, they utilized top-down goals for completing story-writing tasks. While the goals served to effectively accommodate outputs from different crowd workers, one distinct characteristic of this method was that goals were not pre-embedded in the writing system but were chosen among other workers from previous stages. Thus moving around the iterative steps of reflection and revision goals, workers came up with better ideas for given tasks.

\subsubsection{Dynamic Objects and Representations}

As a strategy of employing the objects and representations mechanism, workflows have been dominantly used in the crowdsourcing literature. Workflows serve as an object and representation that reflects the division and sequence of work. In macro-task coordination, because of the nature of macro-tasks- which are often nondecomposable, context-dependent, and contingent on progress and changes-designing workflows has been a challenging problem (Retelny et al. 2017).

Researchers have investigated workflows for macro-tasking that can be collaboratively developed and amenable to work progress. One example is Turkomatic, developed by Kulkarni et al. (2012). The system employs a list view and hierarchical graphs to show the structure of decomposed tasks by workers and the status of each task, whether waiting, in progress, or done. Another example is a sentence-level scaffolding structure that Kim et al. (2017) utilized to define subsequent goals and tasks to accomplish in Mechanical Novel. It helped workers not only generate suggestions for further edits on a draft but also identify goals and tasks at a given stage.

Objects have also been suggested to support workers in decomposing complex tasks. Kaur et al. (2018) introduced a "cognitive scaffold" for crowd workers to plan action items to accomplish complex and context-embedded tasks. Specifically, the researchers provided a vocabulary that comprised possible functions and subtasks based on the analysis of the crowd's comments on possible writing goals. The researchers found it useful for workers to map out writing tasks.

\subsubsection{Roles Loosely Held}

We found several studies employing role-based coordination along with defining hierarchical role structures. Haas et al. (2015) built Argonaut, which automatized control of crowd workers' output and their quality. To review task output and quality effectively, the researchers defined positions of reviewers, reflecting different levels of their review expertise, and made a hierarchical structure of the positions. Using the hierarchy, the researchers identified a pool of trusted workers and assigned them to different positions. Valentine et al. (2017) proposed flash organizations that were flexibly assembled, role-based structures. The hierarchical structure loosely defined roles and responsibilities to help workers use their skills and competence to adjust to the progress of work. This approach allowed for the mobilization of different 
sets of crowd workers depending on their expertise and availability. In addition, to do more efficient substitution, Salehi et al. (2017) addressed the role mechanism by managing familiarity and availability. By creating a loosely bounded team that consisted of crowd workers who had a common understanding of their role and relationship to the project, the researchers could occupy roles with different workers who were available at a given point, and the researchers found that this approach supported complex-task completion.

\subsubsection{Routines}

We found one article that discussed the use of routines as a coordination mechanism. Salehi et al. (2017) noted that routines can be useful when uncertainty and complexity of a problem is low. As they noted, routines can help workers develop common knowledge about how to produce a desired outcome based on prescribed procedures. Salehi et al. discovered that worker familiarity, as routines would accomplish, could lead to better coordination by increasing workers' knowledge of how their teammates worked.

\subsubsection{Proximity}

Our review found one study that employed proximity as familiarity (see Salehi et al. 2017), but none employed proximity as visibility to coordinate macro-tasks. This might be because the studies we reviewed were motivated to tackle problems related to online crowdsourcing, where crowd workers are distributed and rarely have familiarity with one another.

\subsubsection{Summary}

Overall, our review of coordination in the macro-tasking crowdsourcing literature revealed that much of the literature has focused on a small subset of coordination theories. More specifically, we found that macro-tasking studies on coordination have largely focused on establishing plans and rules $(80 \%)$ to describe a final goal and subtasks (see Table 2.2). This was followed by the studies on building objects and representations (50\%). Role-based approaches were also used as a coordination mechanism for macro-tasks in a few studies (40\%). Routines and proximity were discussed in one study. 
Table 2.2 Literature review based on coordination mechanisms

\begin{tabular}{l|c|c|c|c|c}
\hline & Plans and rules & $\begin{array}{l}\text { Objects and } \\
\text { representations }\end{array}$ & Roles & Routines & Proximity \\
\hline $\begin{array}{l}\text { Kittur et al. (2011) } \\
\text { (Crowdforge) }\end{array}$ & $\mathrm{X}$ & $\mathrm{X}$ & & & \\
\hline $\begin{array}{l}\text { Kulkarni et al. } \\
\text { (2012) }\end{array}$ & & $\mathrm{X}$ & & \\
(Turkomatic) & & $\mathrm{X}$ & $\mathrm{X}$ & & \\
\hline $\begin{array}{l}\text { Haas et al. (2015) } \\
\text { (Argonaut) }\end{array}$ & & $\mathrm{X}$ & $\mathrm{X}$ & & \\
\hline $\begin{array}{l}\text { Teevan et al. } \\
\text { (2016) } \\
\text { (Microwriter) }\end{array}$ & $\mathrm{X}$ & $\mathrm{X}$ & $\mathrm{X}$ & & \\
\hline $\begin{array}{l}\text { Kim et al. (2017) } \\
\text { (Mechanical } \\
\text { novel) }\end{array}$ & & $\mathrm{X}$ & $\mathrm{X}$ & & \\
\hline $\begin{array}{l}\text { Retelny et al. } \\
\text { (2017) (No } \\
\text { workflow) }\end{array}$ & & $\mathrm{X}$ & $\mathrm{X}$ & \\
\hline $\begin{array}{l}\text { Salehi et al. (2017) } \\
\text { (Huddler) }\end{array}$ & & & & & \\
\hline $\begin{array}{l}\text { Valentine et al. } \\
\text { (2017) (Flash } \\
\text { organization) }\end{array}$ & & $\mathrm{X}$ & & & \\
\hline $\begin{array}{l}\text { Kaur et al. (2018) } \\
\text { (Vocabulary) }\end{array}$ & & $\mathrm{X}$ & & \\
\hline $\begin{array}{l}\text { Schmitz and } \\
\text { Lykourentzou } \\
\text { (2018) (Task } \\
\text { assignment and } \\
\text { sequencing) }\end{array}$ & & & & & \\
\hline
\end{tabular}

\subsection{Agenda for Future Research}

Based on our brief literature review on coordination theories used in macro-tasking, the stigmergic and relational coordination theories have been studied the least, along with two integrative mechanisms: proximity (visibility and familiarity) and routines. Yet, we believe these theories and mechanisms offer the greatest potential for the crowdsourcing of macro-tasks. First, these theories and mechanisms rely on social processes of interaction along with adjustment to emergent states. They place much less emphasis on a priori definition of interdependencies among tasks or even roles among crowd workers. Approaches that focus on defining work upfront are likely to always rely heavily on requestors. To the contrary, both stigmergic and relational coordination along with proximity (visibility and familiarity) and routines rely more on facilitating the establishment of more informal coordination, which allows for 
more spontaneous coordination of work. We believe these informal coordination approaches are likely to be more effective ways of coordinating crowdsourcing as it becomes increasingly oriented to macro- rather than micro-tasks. In addition, many of the concepts of TMS are embodied in relational coordination's "shared knowledge" concept.

To advance our understanding in the areas of stigmergic and relational coordination, we present and discuss several important research questions. In addition, we present design propositions related to stigmergic and relational coordination. Design propositions are general statements regarding the relationship between a design element and other concepts. In this chapter, design propositions are general statements regarding the relationship between the design of a system and coordination approaches.

\subsubsection{Stigmergic Coordination}

Stigmergic coordination refers to coordination based on traces, without explicit communication among workers (Heylighen 2016; Rezgui and Crowston 2018). Because stigmergic coordination doesn't necessarily require communication among workers and is done instead by interaction between workers and environments, including traces left by other workers, it could be beneficial in coordinating macro-tasks. For example, the stigmergic coordination process doesn't involve setting up plans and controls. This would help crowd workers readily get involved in work and adjust their behaviors to the status and progress of work. Thus, we suggest research questions that could advance macro-task coordination by employing stigmergic approaches.

Research Question 1: How can we support the traces of prior work in the crowdsourcing of macro-tasks?

First, as discussed, traces in stigmergic coordination serve as mediating objects that enable the bridging of the actions of prior workers with those of subsequent workers. Traces help inform workers of both the progress of work and the remaining work. Thus, developing systems that support leaving traces effectively could be one way to support stigmergic coordination for crowdsourcing macro-tasks. For example, crowdsourcing systems could be designed to provide features that help workers leave comments or remarks next to their work. These systems could be designed to include features that track the progress of work and make it salient.

Research Question 2: How can we promote the shared interpretations of traces in the crowdsourcing of macro-tasks?

Workers who engage in stigmergic coordination use traces to implicitly determine what has been done and what to do next. This implicit coordination can occur because the workers belong to a community that has a shared context. This shared context helps to establish common work norms and routines among members of a given community. This is what allows workers to employ traces as a mechanism to engage 
Table 2.3 Design propositions for stigmergic coordination coordination in macro-tasking crowdsourcing. ers to engage in stigmergic coordination to tackle macro-tasks. support recruitment for macro-tasks requiring COBOL. to be selected for macro-tasks.

in implicit coordination. Next, we discuss three approaches to leveraging stigmergic

One approach is to recruit crowd workers who already have a shared context, norms, and routines. This could be done by recruiting groups of workers from existing online communities and peer platforms like GitHub. For example, a group of workers from GitHub could be recruited to work on a macro-tasking project. These workers would already have a shared context, norms, and routines. To leverage their existing shared context, norms, and routines obtained using the GitHub platform, the crowdsourcing platform should be set up similarly to the GitHub platform. Together the workers from the GitHub community and the new crowdsourcing platform that supports the workers' shared context, norms, and routines should allow crowd work-

Another approach is to create an online community from which to recruit crowd workers. This approach offers two advantages. One, it would allow crowd workers to develop a shared context, norms, and routines. Over time, these crowd workers would be able to engage in stigmergic coordination in the same way as crowd workers who are members of current online communities. Two, this approach would allow for the creation of an online community that focuses on a subject or theme that might not exist. For example, imagine if macro-tasks required workers who were familiar with a specific programming language like the common business-oriented language (COBOL). Many mainframes still rely on programs written in this language, although it is not widely taught. Creating an online community of COBOL programmers would

Finally, the third approach is to require crowd workers who want to participate in macro-tasking to have experience working in a specific online community. Potential workers would be directed to participate in a specific online community before they could be eligible to be selected for macro-tasking. This would allow crowd workers the opportunity to learn basic knowledge and rules from an existing online community. Over time they would develop the shared context, norms, and routines needed

Table 2.3 presents a summary of the three design propositions related to stigmergic coordination. Design propositions were derived from the research questions 1 and 2.

Stigmergic coordination design propositions

Design proposition 1: Crowdsourcing systems that support stigmergic coordination will help crowd workers effectively accomplish macro-tasks

Design proposition 1a: To promote stigmergic coordination, crowdsourcing systems must facilitate the leaving and making visible the traces of prior work

Design proposition $1 \mathrm{~b}$ : To promote stigmergic coordination, crowdsourcing systems must facilitate a shared interpretation of the traces of prior work

Design proposition 1c: To promote stigmergic coordination, crowdsourcing systems must support the leveraging of shared work norms and practices 


\subsubsection{Relational Coordination}

Relational coordination theory describes relationship in terms of three dimensions: shared goals, shared knowledge, and mutual respect; and communication in four dimensions: frequency, timeliness, accuracy, and problem-solving focus. These dimensions are both representative of and impacted by the quality of social relationship within a given collective. The benefits of relational coordination are that it allows workers to coordinate complex work in dynamic environments. This is accomplished by allowing individuals to coordinate their efforts by working through problems cooperatively. Relational coordination can be viewed as a set of mechanisms that provide a canvas for a collective set of painters. As long as collectives maintain quality relationships, they can leverage elements of their relationships to effectively coordinate work. In fact, it is this reliance on the quality of relationships that clearly differentiates relational coordination from stigmergic coordination.

Next, we suggest research questions that could advance our understanding of crowdsourcing macro-tasks through relational coordination.

\section{Research Question 3a: How can shared knowledge be promoted in the crowdsourcing of macro-tasks?}

According to relational coordination, shared knowledge helps workers to become aware of their interdependencies with coworkers and of one another's potential contribution to work. This awareness helps to facilitate effective and accurate communication. There are two big challenges with achieving a sufficient level of shared knowledge in crowdsourcing. One, workers engaged in crowdsourcing are often ad hoc and have little prior experience working together. Therefore, they initially have little or no shared knowledge as a group. Two, depending on the amount of time required to complete the task, crowd workers often do not have enough time to develop shared knowledge. Both challenges greatly undermine the ability of crowd workers to rely on shared knowledge as a coordination mechanism.

There are several potential ways to design crowdsourcing systems to promote shared knowledge. First, systems could help crowd workers identify who knows what. This could be done by having a system that publicly displays each worker's profile. This profile could include the worker's educational and work experience. The workers should give consent before profiles are displayed, and more or less information might be displayed based on who is viewing the profile. For example, members of the macrotask team might have access to more information about each worker than members of the public. Second, systems should be designed to help make as much as possible of the individual crowd worker's knowledge explicitly available to all others. This could be done by promoting the sharing, using, and ultimate integration of knowledge across the team (Robert et al. 2008, 2018). Crowdsourcing systems would need to be designed to not only provide both asynchronous and synchronous communication capabilities but several other important features. For example, these systems should make it easy to search the repository of communications, including multichannel communications and use of visual aids such as sketches, snapshots, whiteboards, 
links, documents, and templates (Alavi and Tiwana 2002). These features should also provide real-time editing and commenting so that workers could explain their actions to others as well as inquire about why actions were taken.

\section{Research Question 3b: How can shared goals be leveraged in the crowdsourcing of macro-tasks?}

Shared goals are another important coordination mechanism in relational coordination that can be problematic in crowdsourcing macro-tasks. Shared goals motivate workers to engage in high-quality communication with others. This guides workers to focus more on problem-solving-related communication than emotional and nonproductive communication. On one hand, it should be easy to promote shared goals in the crowdsourcing of macro-tasks. The crowd workers have been assembled to accomplish a specific macro-task. This macro-task is essentially the shared goal. On the other hand, it can be difficult for crowd workers to maintain a shared view on the progress or lack of progress of those shared goals. This can be even more problematic in macro-task work environments, which can be more dynamic than static micro-tasking work environments.

To promote a shared view of goals in the crowdsourcing of macro-tasks, we turn to boundary objects. According to Okhuysen and Bechky (2009), boundary objects are a type of object and representation coordination mechanism. As stated, boundary objects help to communicate problems, ideas, and activities across teams. The biggest benefit of boundary objects is that they allow an individual's specific understanding of a given situation to be framed within the larger context of the collective's situation (Bechky 2003). Therefore, boundary objects can be used to communicate the status of the collective's situation to all members of the collective, without the need for workers to fully understand each member's specific situation. In the case of crowdsourcing macro-tasks, boundary objects could promote a shared view of goals by allowing crowd workers to accomplish individual objectives within the framework of the collective's goals. However, it is not clear which boundary objects should be employed. One option would be to focus on promoting situation awareness.

The promotion of situation awareness offers a viable approach to understanding how to design boundary objects to promote a shared view of goals in macro-tasks. Endsley (1995) formally defined situation awareness as "the perception of the elements in the environment within a volume of time and space, the comprehension of their meaning, and the projection of their status in the near future" (p. 36). A more informal definition is an ability to perceive and comprehend information, which allows for the prediction of future courses of action in a dynamic environment. In the case of crowdsourcing, we define crowdsourcing situation awareness as the ability of crowd workers to perceive and comprehend the status of their crowd's work and to forecast the needed future courses of action to complete the crowd's work. Situation awareness is similar to the use of traces in stigmergic coordination, with several differences. The use of traces in stigmergic coordination is the result of a shared context, norms, and routines obtained in large part by one's socialization into a community. However, situation awareness can be obtained without the need for this 
socialization process, and although it can help to promote a shared context, it may or may not lead to shared norms and routines. In addition, stigmergic coordination occurs through implicit coordination, whereas situation awareness occurs primarily through explicit coordination among members of the collective.

Research Question 3c: How can systems be designed to support situation awareness in the crowdsourcing of macro-tasks?

To accomplish this, scholars should turn to the study of visualization. Visualization is science that focuses on understanding how to best display information to humans. A full review of this research area is beyond the scope of this book chapter, but visualization techniques have been used to reduce cognitive load (Anderson et al. 2011). It is likely that current research on visualization can be leveraged and that new research will also be needed. Questions about how best to support situation awareness specifically for crowdsourcing macro-tasks would need to be addressed. A program of research in this area might attempt to define key attributes of the type of macro-task and crowd workers, and stage of work, and how these factors influence the ways information should be displayed.

Research Question 3d: How can mutual respect be promoted in the crowdsourcing of macro-tasks?

In relational coordination, mutual respect increases the level of receptiveness to communication with others, leading to increased opportunity for improving shared knowledge and solving problems effectively. On one hand, the challenges to achieving mutual respect are the same as those to achieving shared knowledge in crowdsourcing macro-tasks. These include the often ad hoc nature of crowdsourcing, which involves assembling crowd workers with little experience working together and a short duration of time required to complete the task. Some challenges are also different; for example, crowd workers could also develop a mutual disrespect for one another. Each of these challenges could greatly undermine the ability of crowd workers to rely on mutual respect as a coordination mechanism.

To combat these challenges, there are several potential ways to design crowdsourcing systems to promote mutual respect. First, systems could promote mutual respect through trust. This could be done by designing systems that display recommendations from others who have worked with the crowd worker. This system could share positive narratives about the crowd worker's behavior. Such a system could include a peer evaluation that rates crowd workers on their respect for others. Second, systems could be designed to monitor the level of mutual respect among crowd workers. For example, Munson et al. (2014) developed a system that monitored the email communications among teams to determine their degree of trust and respect through linguistic mimicry. Questions around how such systems could measure mutual respect or what data should be used to measure it would need to be further investigated. For example, it is not clear how such measures might be drawn from prior studies or whether new measures better suited to a macro-tasking context need to be identified. Systems like these could be designed to diagnose the level of mutual respect among crowd workers to determine whether interventions are needed. 
Finally, interventions should be designed to help promote mutual respect when needed. Although research is needed to understand the types of interventions necessary, we recommend several potential avenues. The research on conflict and conflict resolution offers a rich set of literature to draw from. For example, this research has identified several types of conflict: relationship, process, and task (Jehn 1997). Relationship conflict is related to personal disagreements among team members, whereas task- and process-focused conflicts are related to work but are not personal disagreements. Research has shown that relationship conflict is always detrimental to performance, whereas task and process conflicts can be beneficial to team performance (Windeler et al. 2015). Systems should be designed to determine which type of conflict is occurring. The literature on conflict resolution has identified several approaches to resolving conflict in groups. These include avoidance, accommodation, competition, collaboration, and compromise (Kankanhalli et al. 2006; MontoyaWeiss et al. 2001; Paul et al. 2004). Although a full review and discussion of each of these are beyond the scope of this chapter, what is clear is that each approach has pros and cons and would likely require different system interventions. A program of research could explore both the effectiveness of each approach in the context of crowdsourcing macro-tasks and how to best design systems to support each approach.

\section{Research Question 3e: What is the most effective way to promote communication in the crowdsourcing of macro-tasks?}

Relational coordination defines communication in four dimensions: frequency, timeliness, accuracy, and problem-solving focus (Gittell 2002, 2006). The easiest and first step toward supporting frequent, timely, accurate, and problem-solving-focused communication is to design crowdsourcing systems that allow effective communications. Features of such systems have been identified in the form of both asynchronous and synchronous communications as well as multichannel communications. However, systems could be designed to go beyond this and take a more active role in several meaningful ways. Systems could be designed to prompt communications. A research agenda could be built on the investigation of the effectiveness of types of prompts. For example, days before a work deadline the system could send an email to everyone inquiring about the status of the group's work. This might encourage crowd workers to engage in task-focused communications about the upcoming deadline. Nudges could also be used to alert crowd workers when the status of their group's work has changed or when crowd workers have left questions for others to answer. Crowdsourcing systems could be set up to require timely status updates that rely on the input of every crowd worker and go out to every crowd worker. A research agenda could also be built on understanding the effectiveness of the content of such messages. For example, research has shown that the framing of messages impacts how people choose to respond or not respond to them (Jung and Mellers 2016). Research should be directed at understanding the best content to promote communication frequency, timeliness, accuracy, and problem-solving focus among crowd workers.

Table 2.4 presents a summary of the four design propositions related to relational coordination. Design propositions were derived from research questions $3 \mathrm{a}, 3 \mathrm{~b}, 3 \mathrm{c}$, $3 \mathrm{~d}$ and $3 \mathrm{e}$. 
Table 2.4 Design propositions for relational coordination

\begin{tabular}{l}
\hline Relational coordination design propositions \\
\hline Design proposition 2: Crowdsourcing systems that support relational coordination will help \\
crowd workers effectively accomplish macro-tasks \\
\hline Design proposition 2a: To promote relational coordination, crowdsourcing systems must \\
facilitate the creating and sharing of collective knowledge \\
\hline Design proposition 2b: To promote relational coordination, crowdsourcing systems must \\
facilitate the creating and sharing of common goals \\
\hline Design proposition 2c: To promote relational coordination, crowdsourcing systems must \\
support the development of mutual respect \\
\hline Design proposition 2d: To promote relational coordination, crowdsourcing systems must \\
facilitate effective communication
\end{tabular}

\subsubsection{Limitations}

In this chapter, we acknowledge that theories of coordination have shared or overlapping concepts. Nonetheless, for the most part, we treated them as separate and distinct when discussing their pros and cons. Our separation of each theory of coordination might at times have been more artificial and arbitrary. Scholars studying issues related to crowdsourcing coordination should consider hybrid approaches that combine various elements of each theory. For example, stigmergic coordination could be augmented with role-based coordination. This could be accomplished by bringing in outsiders unfamiliar with the work norms and practices and defining a specific role for them in the work structure. By defining their role, work disruption resulting from their lack of familiarity with traces should be kept at a minimum. We also acknowledge that each theory has its own rich and insightful literature that goes beyond the scope of this one chapter. This chapter provides a brief introduction of each theory. Where brevity and conciseness end and confusion and incompleteness begin is often debatable. That being the case, the goal of this chapter was to draw attention to the issues related to coordinating macro-tasking in crowdsourcing environments. Our recommendations are but suggestions and readers are advised to dig deeper into these issues themselves. Finally, we provide design propositions that link theory to design elements. Our propositions, like all propositions, are general statements. Ultimately, hypotheses should be derived from our design propositions before they can be empirically tested. This is a challenge we hope future scholars choose to undertake.

\subsection{Conclusions}

Crowdsourcing macro-tasking places more emphasis on coordinating complex, interdependent, and less decomposable tasks. This chapter reviewed and recommended several theories of coordination to address issues related to coordinating macro-tasks. 
It presented a research agenda and design propositions for each recommended theory of coordination. The research agendas and design propositions are far from complete, and more work is needed with regard to both theoretical development and empirical verification. Nonetheless, we hope this chapter is the first step in advancing our understanding of crowdsourcing coordination used for macro-tasks.

Acknowledgements This book chapter was supported in part by the National Science Foundation [grant CHS-1617820].

\section{References}

Adler, P. S., Kwon, S. W., \& Heckscher, C. (2008). Perspective-professional work: The emergence of collaborative community. Organization Science, 19(2), 359-376.

Alavi, M., \& Tiwana, A. (2002). Knowledge integration in virtual teams: The potential role of KMS. Journal of the American Society for Information Science and Technology, 53(12), 1029-1037.

Anderson, E. W., Potter, K. C., Matzen, L. E., Shepherd, J. F., Preston, G. A., \& Silva, C. T. (2011). A user study of visualization effectiveness using EEG and cognitive load. In Computer graphics forum (Vol. 30, No. 3, pp. 791-800). Oxford, UK: Blackwell Publishing Ltd.

Andres, H. P., \& Zmud, R. W. (2002). A contingency approach to software project coordination. Journal of Management Information Systems, 18(3), 41-70.

Austin, J. R. (2003). Transactive memory in organizational groups: The effects of content, consensus, specialization, and accuracy on group performance. Journal of Applied Psychology, 88(5), 866-878.

Bechky, B. A. (2003). Sharing meaning across occupational communities: The transformation of understanding on a production floor. Organization Science, 14(3), 312-330.

Bechky, B. A. (2006). Gaffers, gofers, and grips: Role-based coordination in temporary organizations. Organization Science, 17(1), 3-21.

Bolici, F., Howison, J., \& Crowston, K. (2009). Coordination without discussion? Socio-technical congruence and stigmergy in free and open source software projects. Paper presented at the International Conference on Software Engineering, Vancouver, BC, Canada. Retrieved from http://citeseerx.ist.psu.edu/viewdoc/download?doi=10.1.1.193.7473\&rep=rep1\&type=pdf.

Bolici, F., Howison, J., \& Crowston, K. (2016). Stigmergic coordination in FLOSS development teams: Integrating explicit and implicit mechanisms. Cognitive Systems Research, 38, 14-22.

Brandon, D. P., \& Hollingshead, A. B. (2004). Transactive memory systems in organizations: Matching tasks, expertise, and people. Organization Science, 15(6), 633-644.

Choi, S. Y., Lee, H., \& Yoo, Y. (2010). The impact of information technology and transactive memory systems on knowledge sharing, application, and team performance: A field study. MIS Quarterly, 34(4), 855-870.

Crowston, K. (1994). A taxonomy of organisational dependencies and coordination mechanisms. MIT Center for Coordination Science Working Paper. Massachusetts Institute of Technology, August 1994.

Crowston, K., Howison, J., \& Rubleske, J. (2006). Coordination theory: A ten year retrospective. In P. Zhang \& D. Galletta (Eds.), Human-computer interaction in management information systems-foundations (pp. 120-138). Armonk, NY: M. E. Sharpe Inc.

Endsley, M. R. (1995). Toward a theory of situation awareness in dynamic systems. Human Factors, 37(1), 32-64.

Faraj, S., \& Xiao, Y. (2006). Coordination in fast-response organizations. Management Science, $52(8), 1155-1169$. 
Feldman, M. S. (2000). Organizational routines as a source of continuous change. Organization Science, 11(6), 611-629.

Gittell, J. H. (2002). Coordinating mechanisms in care provider groups: Relational coordination as a mediator and input uncertainty as a moderator of performance effects. Management Science, 48(11), 1408-1426.

Gittell, J. H. (2006). Relational coordination: Coordinating work through relationships of shared goals, shared knowledge and mutual respect. In O. Kyriakidou \& M. F. Özbilgin (Eds.), Relational perspectives in organizational studies: A research companion (pp. 74-94). Cheltenham, UK: Edward Elgar Publishers.

Gittell, J. H. (2011). New directions for relational coordination theory. In K. S. Cameron \& G. M. Spreitzer (Eds.), The Oxford handbook of positive organizational scholarship (pp. 400-411). New York, NY: Oxford University Press.

Gittell, J. H. (2016). Transforming relationships for high performance: The power of relational coordination. Palo Alto, CA: Stanford University Press.

Haas, D., Ansel, J., Gu, L., \& Marcus, A. (2015). Argonaut: Macrotask crowdsourcing for complex data processing. Proceedings of the VLDB Endowment, 8(12), 1642-1653.

Heylighen, F. (2015). Stigmergy as a universal coordination mechanism: Components, varieties and applications. In T. Lewis \& L. Marsh (Eds.), Human stigmergy: Theoretical developments and new applications. New York, NY: Springer.

Heylighen, F. (2016). Stigmergy as a universal coordination mechanism I: Definition and components. Cognitive Systems Research, 38, 4-13.

Holland, O., \& Melhuish, C. (1999). Stigmergy, self-organization, and sorting in collective robotics. Artificial Life, 5(2), 173-202.

Howe, J. (2006). The rise of crowdsourcing. Wired, 14(6), 1-4.

Jehn, K. A. (1997). A quantitative analysis of conflict types and dimensions in organizational groups. Administrative Science Quarterly, 42(3), 530-557.

Jung, J. Y., \& Mellers, B. A. (2016). American attitudes toward nudges. Judgment \& Decision Making, 11(1), 62-74.

Kankanhalli, A., Tan, B. C., \& Wei, K. K. (2006). Conflict and performance in global virtual teams. Journal of Management Information Systems, 23(3), 237-274.

Kaur, H., Williams, A. C., Thompson, A. L., Lasecki, W. S., Iqbal, S. T., \& Teevan, J. (2018). Creating better action plans for writing tasks via vocabulary-based planning. Proceedings of the ACM on Human-Computer Interaction, 2(CSCW), 86.

Khuong, A., Gautrais, J., Perna, A., Sbaï, C., Combe, M., Kuntz, P., et al. (2016). Stigmergic construction and topochemical information shape ant nest architecture. Proceedings of the National Academy of Sciences, 113(5), 1303-1308.

Kim, J., Sterman, S., Cohen, A. A. B., \& Bernstein, M. S. (2017). Mechanical novel: Crowdsourcing complex work through reflection and revision. In Proceedings of the 2017 ACM Conference on Computer-supported Cooperative Work and Social Computing (pp. 233-245). New York, NY: ACM.

Kittur, A., Smus, B., Khamkar, S., \& Kraut, R. E. (2011). Crowdforge: Crowdsourcing complex work. In Proceedings of the 24th annual ACM symposium on User Interface Software and Technology (pp. 43-52). New York, NY: ACM.

Kulkarni, A., Can, M., \& Hartmann, B. (2012). Collaboratively crowdsourcing workflows with Turkomatic. In Proceedings of the ACM 2012 Conference on Computer-supported Cooperative Work (pp. 1003-1012). New York, NY: ACM.

Lave, J. (1991). Situating learning in communities of practice. Perspectives on Socially Shared Cognition, 2, 63-82.

Lave, J. (2009). The practice of learning. In K. Illeris (Ed.), Contemporary learning theories (pp. 200-208). London, UK: Routledge.

Lave, J., \& Wenger, E. (1991). Situated learning: Legitimate peripheral participation. New York, NY: Cambridge University Press. 
Lewis, K. (2003). Measuring transactive memory systems in the field: Scale development and validation. Journal of Applied Psychology, 88, 587-604.

Malone, T. W., \& Crowston, K. (1994). The interdisciplinary study of coordination. ACM Computing Surveys (CSUR), 26(1), 87-119.

Marques-Quinteiro, P., Curral, L., Passos, A. M., \& Lewis, K. (2013). And now what do we do? The role of transactive memory systems and task coordination in action teams. Group Dynamics: Theory, Research, and Practice, 17(3), 194-206.

Montoya-Weiss, M. M., Massey, A. P., \& Song, M. (2001). Getting it together: Temporal coordination and conflict management in global virtual teams. Academy of Management Journal, 44(6), $1251-1262$.

Moreland, R. L. (1999). Transactive memory: Learning who knows what in work groups and organizations. In L. L. Thompson, J. M. Levine, \& D. M. Messick (Eds.), Shared cognition in organizations: The management of knowledge (pp. 3-31). Mahwah, NJ: Erlbaum.

Munson, S. A., Kervin, K., \& Robert Jr., L. P. (2014). Monitoring email to indicate project team performance and mutual attraction. In Proceedings of the 17th ACM Conference on Computersupported Cooperative Work \& Social Computing (pp. 542-549). New York, NY: ACM.

Okhuysen, G. A., \& Bechky, B. A. (2009). Coordination in organizations: An integrative perspective. In J. P. Walsh \& A. P. Brief (Eds.), Academy of management annals (Vol. 3, pp. 463-502). Essex, UK: Routledge.

Paul, S., Seetharaman, P., Samarah, I., \& Mykytyn, P. P. (2004). Impact of heterogeneity and collaborative conflict management style on the performance of synchronous global virtual teams. Information \& Management, 41(3), 303-321.

Ren, Y., \& Argote, L. (2011). Transactive memory systems 1985-2010: An integrative framework of key dimensions, antecedents, and consequences. Academy of Management Annals, 5(1), 189-229.

Retelny, D., Bernstein, M. S., \& Valentine, M. A. (2017). No workflow can ever be enough: How crowdsourcing workflows constrain complex work. Proceedings of the ACM on Human-Computer Interaction, $1(\mathrm{CSCW}), 89$.

Retelny, D., Robaszkiewicz, S., To, A., Lasecki, W. S., Patel, J., Rahmati, N.,... Bernstein, M. S. (2014). Expert crowdsourcing with flash teams. In Proceedings of the 27th annual ACM Symposium on User Interface Software and Technology (pp. 75-85). New York, NY: ACM.

Rezgui, A., \& Crowston, K. (2018). Stigmergic coordination in Wikipedia. In Proceedings of the 14th International Symposium on Open Collaboration (pp. 1-12). Paris, France: ACM Press.

Robert, L. P. (2016). Far but near or near but far?: The effects of perceived distance on the relationship between geographic dispersion and perceived diversity. In Proceedings of the $2016 \mathrm{CHI}$ Conference on Human Factors in Computing Systems (pp. 2461-2473). New York, NY: ACM.

Robert, L. P., Dennis, A. R., \& Ahuja, M. (2008). Social capital and knowledge integration in digitally enabled teams. Information Systems Research, 19(3), 314-334. http://pubsonline.informs. org/doi/abs/10.1287/isre.1080.0177.

Robert, L. P., Dennis, A. R., \& Ahuja, M. (2018). Differences are different: Examining the effects of communication media on the impacts of racial and gender diversity in decision-making teams. Information Systems Research, 29(3), 525-545. https://doi.org/10.1287/isre.2018.0773.

Robert, L. P., \& Romero, D. M. (2015). Crowd size, diversity and performance. In Proceedings of the 33rd Annual ACM Conference on Human Factors in Computing Systems (pp. 1379-1382). New York, NY: ACM.

Robert, L. P., Jr., \& Romero, D. M. (2017). The influence of diversity and experience on the effects of crowd size. Journal of the Association for Information Science and Technology, 68(2), 321-332.

Salehi, N., McCabe, M., Valentine, M., \& Bernstein, M. S. (2017). Huddler: Convening stable and familiar crowd teams despite unpredictable availability. In Proceedings of the 20th ACM Conference on Computer-supported Cooperative Work \& Social Computing (CSCW'17). New York, NY: ACM.

Schmitz, H., \& Lykourentzou, I. (2018). Online sequencing of non-decomposable macrotasks in expert crowdsourcing. ACM Transactions on Social Computing, 1(1), 1-34. 
Strode, D. E., Huff, S. L., Hope, B., \& Link, S. (2012). Coordination in co-located agile software development projects. Journal of Systems and Software, 85(6), 1222-1238.

Suchman, L. (1987). Plans and situated actions: The problem of human-machine communication. Cambridge, MA: Cambridge University Press.

Teevan, J., Iqbal, S. T., \& Von Veh, C. (2016). Supporting collaborative writing with microtasks. In Proceedings of the 2016 CHI Conference on Human Factors in Computing Systems (pp. 2657-2668). New York, NY: ACM.

Valckenaers, P., Kollingbaum, M., \& Van Brussel, H. (2004). Multi-agent coordination and control using stigmergy. Computers in Industry, 53(1), 75-96.

Valentine, M. A., \& Edmondson, A. C. (2014). Team scaffolds: How mesolevel structures enable role-based coordination in temporary groups. Organization Science, 26(2), 405-422.

Valentine, M. A., Retelny, D., To, A., Rahmati, N., Doshi, T., \& Bernstein, M. S. (2017, May). Flash organizations: Crowdsourcing complex work by structuring crowds as organizations. In Proceedings of the 2017 CHI Conference on Human Factors in Computing Systems (pp. 3523-3537). New York, NY: ACM.

Wegner, D. M. (1987). Transactive memory: A contemporary analysis of the group mind. In B. Mullen \& G. R. Geothals (Eds.), Theories of group behavior (pp. 185-208). New York, NY: Springer.

Windeler, J., Maruping, L., Robert, L. P., \& Riemenschneider, C. (2015). E-identity, conflict and shared understanding in distributed teams. Journal of the Association for Information Systems, 16(7), 608-645. 